\title{
Association between Piscine Mycobacteriosis and Morgellons Disease: A Review of Literature
}

\section{Buddhini N. Dolapihilla1 (으, Gangoda Elapathage Dulara Mahen Elapatha ${ }^{1}{ }^{\circledR}$, Jase Grimm², Udula Vidanage 3 (1)}

${ }^{1}$ Faculty of Medicine, University of Colombo, Colombo, Sri Lanka

${ }^{2}$ University of the People, Pasadena, USA

${ }^{3}$ Postgraduate Institute of Medicine, University of Colombo, Colombo, Sri Lanka

Email: buddhinin1919@gmail.com, udula.vidanage@gmail.com

How to cite this paper: Dolapihilla, B.N., Elapatha, G.E.D.M., Grimm, J. and Vidanage, U. (2021) Association between Piscine Mycobacteriosis and Morgellons Disease: A Review of Literature. Advances in Infectious Diseases, 11, 320-332.

https://doi.org/10.4236/aid.2021.114029

Received: August 30, 2021

Accepted: October 10, 2021

Published: October 13, 2021

Copyright $\odot 2021$ by author(s) and Scientific Research Publishing Inc. This work is licensed under the Creative Commons Attribution International License (CC BY 4.0).

http://creativecommons.org/licenses/by/4.0/

\begin{abstract}
Piscine mycobacteriosis is a fatal fish illness that affects a variety of species globally. It affects over 200 species of freshwater and marine fish. Several species of Mycobacterium are responsible among them Mycobacterium marinum is the comment. It also affects humans when exposed to contaminated water. In fishes, the symptoms include eroded fins, body surface coated with heavy mucus, changing pigmentation, swelling of abdomen, ulcerative dermal necrosis, and scale loss. In humans, the infection is classified into three clinical groups. Type I is a self-limiting, verruca lesion. Type II is single or numerous subcutaneous granulomas in the presence or absence of ulceration. Type III is deep infections of the tenosynovium, bones, bursa, or joints, resulting in tenosynovitis, osteomyelitis, and septic arthritis. The diagnosis is made by Ziehl-Neelsen acid-fast staining, culture, biochemical reaction, and PCR being the most reliable approach. Piscine mycobacteriosis is treated by antibiotics and vaccination has been considered for its long-term prevention in order to reduce morbidity and mortality. Morgellons disease (MD) is a filamentous dermopathy in which lesions with strange filamentous inclusions appear out of nowhere. Furthermore, formication may accompany dermopathy. The identification of Borrelia spirochetes directly in Morgellons disease patient specimen is constant and repeatable when sensitive and precise detection techniques are utilized. It has been diagnosed by microscopy, histology and molecular diagnostic techniques which are highly sensitive and specific. Morgellons disease is still a myth therefore its treatment is evolving, up to date it has been treated symptomatically.
\end{abstract}

\section{Keywords}

Piscine Mycobacteriosis, Fish Tuberculosis, Mycobacterium marinum, Morgellons Disease, Borrelia Infection 


\section{Introduction}

Piscine mycobacteriosis (fish tuberculosis) is a zoonotic illness that affects both freshwater and marine fish globally. More than 20 strains of Mycobacterium species have been found in fish, although the pathogenesis of the majority of them is unclear [1]. Fish tuberculosis is caused by Mycobacterium marinum, and Mycobacterium fortuitum [2]. According to Runyon's classification, Mycobacterium marinum is an environmental, waterborne aerobic bacterium that belongs to the photochromogenic Group I non-tuberculous Mycobacteria [3]. Mycobacterium marinum is an opportunistic infection responsible for tuberculosis-like disease in fish and can infect people when damaged skin is exposed to a polluted aquatic environment. In immunocompetent patients, the infection manifests as a nodular granulomatous illness that can spread along the lymphatic system's distribution and is generally restricted to the skin and soft tissues [4]. Morgellons disease $(\mathrm{MD})$ is a skin disorder in which lesions with strange filamentous inclusions and/or projections appear out of nowhere. Furthermore, formication (feeling of something crawling across or underneath skin) may accompany the dermopathy [5]. Borrelia spirochetes causative agent of Lyme disease such as $B$. sensu lato (Bbsl), Bb sensu stricto (Bbss), Relapsing Fever Borrelia (RFB) and Borrelia burgdorferi $(\mathrm{Bb})$ species, have been found in Morgellons disease patient's bodily fluids and/or tissue samples. Other infections found in Morgellons disease patient's tissues include Helicobacter pylori (Hp), Treponema denticola, Bartonella henselae, and Rickettsiae species, suggesting that these pathogens may be involved in the development of the dermopathy [6]. The infectious agent in the majority of the Morgellon skin specimens examined was Borrelia burgdorferi sensu stricto. Some other Borrelia species have been found in Morgellons disease patients' skin samples such as Borrelia garinii, Borrelia hermsii and Borrelia miyamotoi [7].

\section{Piscine Mycobacteriosis}

\subsection{Epidemiology}

Piscine mycobacteriosis is a widespread illness of marine, freshwater, and brackish fish that affects over 200 species of freshwater and marine fish throughout a large area that ranges from the subarctic to the tropics. This illness affects tropical aquarium fish as well, and it is a leading cause of morbidity and mortality in free-living fish [3].

\subsection{History and Classification}

Piscine mycobacteriosis is a dangerous and sometimes fatal fish illness that affects a variety of species worldwide [8]. Several species of the genus Mycobacterium are responsible. Mycobacterium species are members of the Mycobacteriaceae family, which is part of the Actinomycetales order. Despite their mycolic cell wall's poor fixation of the Gram stain, these aerobic, non-motile pleomorphic bacilli are Gram+ve and are generally stained with Zeihl-Neelsen method. 
In 1897 Mycobacterium specie was initially isolated from granulomatous lesions in common carp (Cyprinus carpio), which led to the first report of the bacteria in fish. This bacterium was given the name Mycobacterium piscium, and it has subsequently been found in frogs and other animals [9]. In 1903 Mycobacterium chelonae was initially discovered from two sea turtles (Chelona corticata) with pulmonary illness [10]. Mycobacterium fortuitum was discovered from neon tetra (Paracheirodon innesi) in 1953 [11]. In ornamental fish, M. avium subspecies hominissuis is found. In Netherlands $M$. avium was recently isolated in public aquarium from an epaulette shark [12]. Mycobacterium stephanolepidis, novel Mycobacterium specie, was discovered in the thread sail filefish (Stephanolepis cirrhifer) in 2017 [13]. Four Mycobacterium species (M. marinum, M. chelonae, $M$. fortuitum and $M$. gordonae) dominate the clinical landscape. Mycobacterium platypoecilus, Mycobacterium balnei, and Mycobacterium anabanti are now known as Mycobacterium marinum. Mycobacterium ranae has been categorized as $M$. fortuitum, whereas Mycobacterium runyonii and Mycobacterium borstelence have been classified as $M$. chelonae species [14]. Table 1 shows the aquatic host and environment of Mycobacterium species responsible for piscine mycobacteriosis [14].

\subsection{Etiology}

Table 1. Mycobacterium species responsible for piscine mycobacteriosis (Delghandi, M.R., et al., 2020) [14].

\begin{tabular}{|c|c|c|}
\hline Species & Aquatic Host & Environment \\
\hline M. septicum & $\begin{array}{l}\text { Zebrafish (Danio rerio), Cichlid } \\
\text { (Pseudotopheus lombardoi), Koi fish }\end{array}$ & Fresh water \\
\hline M. peregrinum & $\begin{array}{l}\text { Labidochromis caeruleus, Black mollies } \\
\text { (Poecilia sphenops), guppies (Poecilia reticulata), } \\
\text { green swordtails (Xiphophorus hellerii), catfish } \\
\text { (Pangasius hypophthalmus) }\end{array}$ & Fresh water \\
\hline M. chelonae & Multiple & Fresh and marine \\
\hline M. avium & Dwarf Cichlid (Apistogramma cacatuodes) & Fresh water \\
\hline M. haemophilum & Zebrafish (Danio rerio) & Fresh water \\
\hline M. lentiflavum & Swordtail (Xiphophorus hellerii) & Fresh water \\
\hline M. gordonae & $\begin{array}{l}\text { Goldfish (Carassius auratus), Guppy (Poecilia } \\
\text { reticulate), Angel fish (Pterophyllum scalare) }\end{array}$ & Marine water \\
\hline M. chesapeaki & Striped bass (Morone saxatilis) & Marine water \\
\hline M. marinum & Multiple & Fresh and marine \\
\hline M. fortuitum & Neon tetra (Paracheirodon innesi) & Fresh water \\
\hline M. pseudoshottsii & $\begin{array}{l}\text { Yellow tail (Seriola quinqueradiata), Greater } \\
\text { amberjack (Seriola dumerili), Striped jack } \\
(\text { Pseudocaranx dentex) }\end{array}$ & Marine water \\
\hline M. shottsii & Striped bass (Morone saxatilis) & Fresh water \\
\hline
\end{tabular}




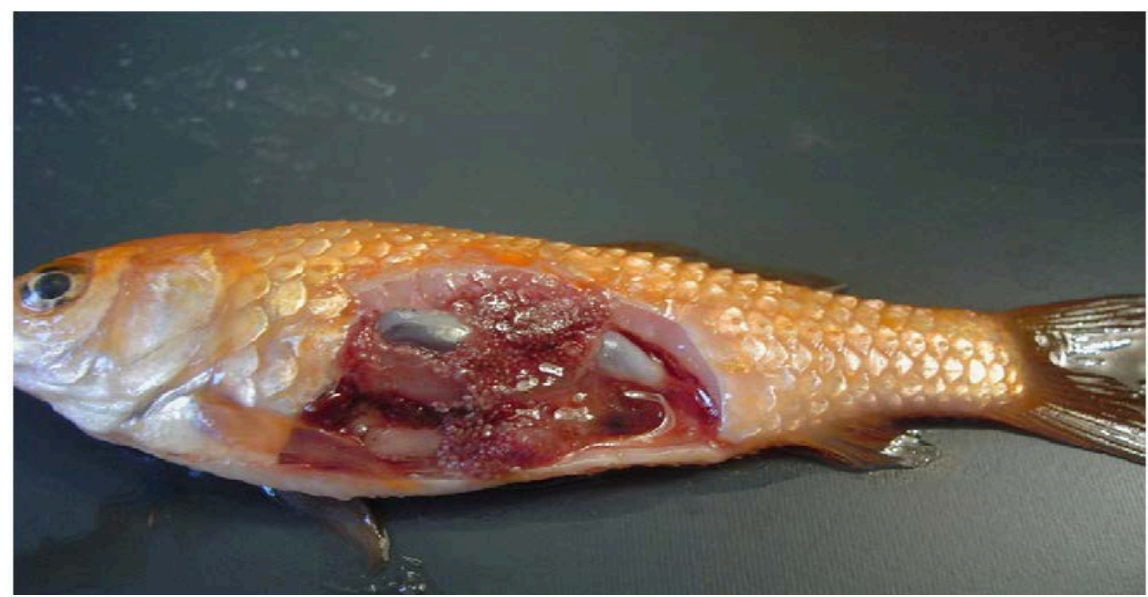

Figure 1. White tubercles present in internal organs (Passantino, A., et al., 2008) [15].

\subsection{Clinical Signs and Symptoms}

The first outward signs include eroded fins and tail rot. Body surface coated with heavy mucus, as well as a changing pigmentation and bleaching have been observed, nonspecific outward symptoms that are frequently quite similar to those of other diseases, such as swelling of abdomen, ulcerative dermal necrosis, and scale loss. White tubercles within internal organs are also seen, as shown in Figure 1 [15]. There have also been reports of external red lesions on the lateral line and shallow asymmetrical ulcers. Exophthalmia, vision loss, pale gills and ascites as well as skeletal abnormalities including spinal curvature or restricted growth, aberrant behavior such as lethargy, poor appetite, and the resultant emaciation, can also be seen [14].

In humans, infections are classified into three clinical groups that have historically been used to guide therapy. Type I is a self-limiting, verruca lesion. Type II is single or numerous subcutaneous granulomas in the presence or absence of ulceration. Type III is deep infections of the tenosynovium, bones, bursa or joints, resulting in tenosynovitis, osteomyelitis and septic arthritis. The lesions are generally $1-2.5 \mathrm{~cm}$ in diameter after 3 - 5 weeks of infection. The spread of lesions is in a proximal manner along the course of the lymphatics is a typical characteristic, albeit it seldom causes axillary discomfort or lymphadenopathy. It is recognized that the illness is rapidly progressing [16].

\subsection{Methodology}

\section{Ziehl-Neelsen acid-fast stain}

After staining with carbolfuchsin, mycobacterial cell wall is resistant to acid-alcohol decolorization. Thus Ziehl-Neelsen acid-fast stain is used to stain fish tissue sections to examine the presence of mycobacteria [14].

\section{Isolation and Culture:}

Mycobacteria are picky and slow growing, and they are prone to be overtaken by rapidly growing organisms on non-selective media. Löwenstein-Jensen (solid medium), Middlebrook 7H10, Middelbrook 7H9 (broth medium), Dorset egg 
media and Petragnani agar are examples of these. Mycobacterium is also resistant to acid and basic compounds, as well as other combinations like benzalkonium chloride and hypochlorite [17].

Depending on the species, Mycobacterium species can be cultured at room temperature or at ambient temperature, and clear colonies can take somewhere between 2 to 28 days to develop [18]. Mycobacterium marinum, for instance, will grow at 30 degrees Celsius, but Mycobacterium shottsii and Mycobacterium pseudoshottsii will grow at 23 degrees Celsius [19]. Mycobacterium salmonifilum may develop at $20^{\circ} \mathrm{C}-30^{\circ} \mathrm{C}$ on particular medium, and after $4-6$ days, smooth colonies can be seen [20]. Mycobacterium haemophilum can be cultured at 29 degree Celsius on Middlebrook 7H10 agar [21].

The interference of Mycobacterium growth on conventional medium complicates culture-based identification of Mycobacterium species from fish skin or gills due to the background presence of microbiota.

\section{Biochemical reaction:}

Numerous biochemical tests are used to identify non tuberculous mycobacteria, this includes Arylsufatase test which is negative and becomes weak positive after 3 days, Nitrate reduction test which is also negative, Catalase test is positive, doesn't grow in the presence of $5 \% \mathrm{NaCl}$ and Urease is positive [14].

\section{Molecular Diagnosis:}

For detection and identification of Mycobacterium spp. that pose a public health risk, a number of molecular diagnostic techniques have been developed. The small subunit, 16S rRNA gene is a preferred target for precise diagnosis, Owing to the accessibility of Mycobacterium $16 \mathrm{~S}$ gene sequences in archives like as GenBank and the Ribosomal Differentiation Database of Microorganisms. In 1997, restriction fragment length polymorphism analysis of the 16S rRNA gene (PCR-RFLP) was used to identify Mycobacterium marinum, Mycobacterium fortuitum, and Mycobacterium chelonae [22]. DNA probe and gene sequencing have also been developed to evaluate Mycobacterium to the species level [23] [24].

When Mycobacteria culture fails, direct sequencing from fish tissues serves as a good alternative [25]. FRET (fluorescence resonance energy transfer) has recently been shown to have excellent specificity when used in conjunction with melting curve analysis to successfully differentiate and identify Mycobacterium marinum from other mycobacteria [26]. A first PCR using SYBR green (at a detection limit of 102 Mycobacterium DNA copies) and then a secondary amplification utilizing FRET in real-time PCR describe this diagnostic approach. Figure 2 shows Micobacterium phlei colonies in Middlebrook agar [14].

\subsection{Treatment}

\section{Antibiotic Therapy:}

M. marinum infection must be treated properly for a quick recovery and to prevent the infection from spreading to deeper tissues and causing permanent 


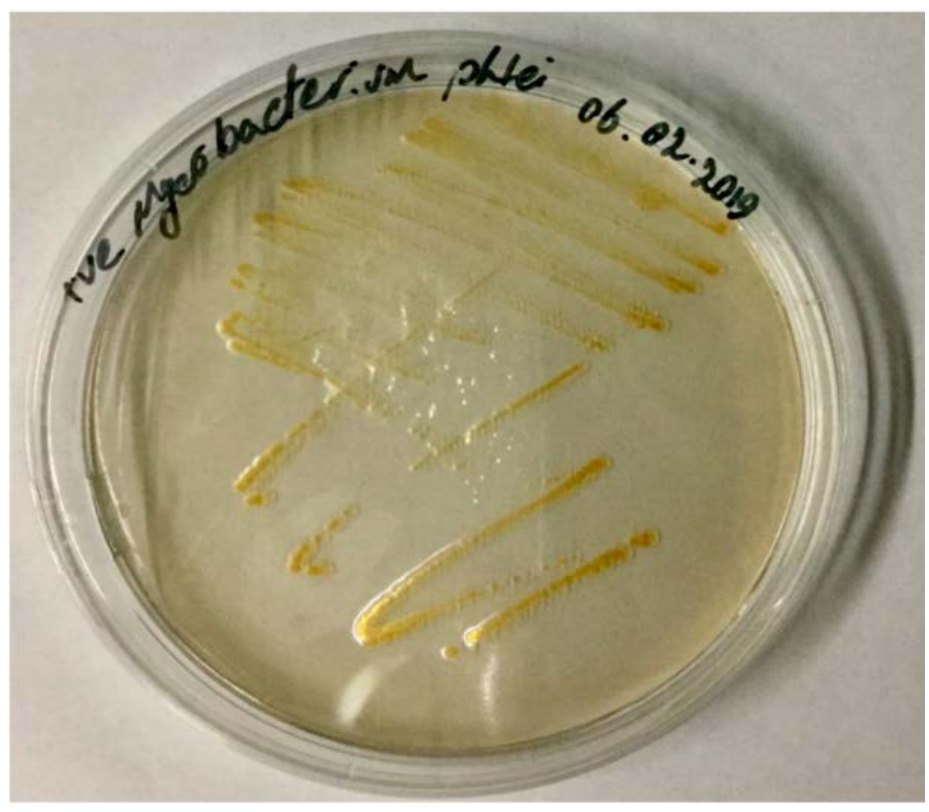

Figure 2. Mycobacterium phlei colonies on Middlebrook 7H10 agar (Delghandi, M.R., et al., 2020) [14].

damage to them. Antibiotics such as rifampicin, erythromycin, streptomycin, ethambutol, kanamycin, isoniazid, minocycline, doxycycline, ethionamide and tetracycline have been used to treat Mycobacteriaceae infections in the past, with some effectiveness [27]. Furthermore, acquired antibiotic resistance is a problem in most genera of harmful bacteria. Mycobacterium marinum strains (AR103K, OR932, TG19, and ATCC927) resistant to sulfamethoxazole and trimethoprim were identified from zebra fish [28].

In humans, monotherapy with clarithromycin, ciprofloxacin and trimethoprim was found to be beneficial in treating superficial cutaneous infections, whereas combination therapy with two medicines may be more successful in treating deeper infections. Combination therapy i.e., rifampicin and ethambutol are indicated in the case of sporotrichoid distribution pattern. Mycobacterium marinum is more resistant to streptomycin, pyrazinamide, and isoniazid therefore they shouldn't be included in the treatment regimen. Several other therapeutic options have been documented and recommended, which includes electrodessication, treatment with X-ray, photodynamic therapy, cryotherapy and local hyperthermic therapy [17].

\section{Vaccination:}

Vaccination for fish mycobacteriosis would be extremely beneficial in terms of illness prevention and control, and numerous attempts have been made throughout the years. In the Japanese flounder ( $P$. olivaceus), the BCG vaccination (Bacillus Calmette and Guerin) was shown to boost the production of numerous immune genes, including IL-1, IL-6, IFN, and TNF- $\alpha$, and this vaccination was linked to improved survival after exposure [29].

Injecting European Seabass (Dicentrarchus labra) with heat killed Mycobacte- 
rium marinum causes the production of IgM and TNF- $\alpha$, which is associated with lower fatality from mycobacteriosis in fish [30].

In zebrafish, the infusion of heat killed Mycobacterium bovis provided cross-protection. Furthermore, immunizing fish with the mycobaterial enzyme RpfE increased the survival rate of fish when high dose of Mycobacterium marinum was injected intraperitoneally [31].

DNA vaccines targeting Mycobacterium spp. Ag85A secreted fibronectin-binding protein have been developed, and hybrid-striped bass (Morone saxatilis Morone chrysops) have been protected against Mycobacterium species, including Mycobacterium marinum. The establishment of an immune response against Mycobacterium marinum by using a DNA vaccine can lower the fatality of vaccinated fish. Furthermore, in zebrafish, the administration of a live attenuated Mycobacterium marinum mutant (L1D) resulted in improved survival greater than $70 \%$ survival rate after 50 days following Mycobacterium marinum injection [14].

\section{Morgellons Disease}

\subsection{History and Background}

Morgellons disease is a rising dermopathy with a global reach. In the 17th century, Sir Thomas Browne identified an illness in French children and named it "Morgellons". It was noticed that the backs of these children had "coarse hairs" sprouting from them. In 1674, Sir Thomas Browne described cutaneous hair like extrusions accompanied by movement sensations [32]. Ekbom's foundational description of delusional parasitosis with perception of insects crawling on or beneath the skin along with feeling of biting and stabbing was seen. Patients would occasionally bring specimens to show doctors in such situations, and these collections comprised of "tiny hairs, grains of sand, little threads and skin scales." He noticed that there were no constant mental disorders evident, aside from delusional ideas of infestation was published in 1938 [7].

\subsection{Etiology}

In recent times Spirochetal infection was first suggested as an etiologic cause for Morgellons disease in 2006. The identification of Borrelia spirochetes directly in Morgellons disease patient specimen is constant and repeatable when sensitive and precise detection techniques are utilized. Even though spirochetal infection appears to be the most important etiologic component in Morgellon disease progression, Morgellons disease patients have a high prevalence of various co-infections including tick-borne illnesses, with Babesia infection being the most common. Other microorganisms have been found directly in Morgellons disease skin specimens, although they have been found less frequently and reliably than Borrelia species. The co-infecting Rickettsia and Bartonella henselae as well as the common human pathogens $H$. pylori and $T$. denticola, are among these pathogens [5]. 


\subsection{Clinical Features}

Clinical signs and symptoms include poor or non-healing dermal lesions, pruritus, sprouting of fibers or excretion of solid particles from the skin; other distressing cutaneous perceptions include stinging, needles, biting sensations and formication. Chronic and recurring symptoms are the most common descriptions for these symptoms [33]. Figure 3 shows multiple lesions in hands and buttocks of a Morgellons patient [7]. Fatigue, trouble concentrating, short-term memory loss, and a low mood are all other symptoms associated with Morgellons disease. Some people have chronic fatigue syndrome, neurocognitive impairments, fibromyalgia, neurological diseases including multiple sclerosis, and mental problems as co-morbid conditions [34]. Healthcare practitioners commonly and incorrectly identify the illness as a type of delusional infestation (DI) or the heritage names delusional parasitosis (DP) and delusions of parasitosis (DOP) due to the false idea that the fibers are generated from textiles [5].

\subsection{Classification}

A classification system for Morgellons disease lesions that takes into account the duration and site of the lesions have been proposed:

> Early localized: Lesions or fibers that have been present for $>3$ months and are limited to a single region i.e. (head, trunk, and extremities).

Early disseminated: Lesions or fibers that have been present for $>3$ months and affect more than one part of the body are considered early disseminated.

Late localized: Lesions or fibers that have been present for $<6$ months and are restricted to a single part of the body.

Late disseminated: Lesions or fibers that have been present for $<6$ months and affect more than one part of the body are classified as late disseminated [7].

\subsection{Classification}

Borrelia spirochetes have been found in skin and body fluid samples from Morgellons disease patients.

\section{Histology and Microscopy:}

Morgellons disease filaments are not textile fibers, according to histological investigations. Biofilaments of human cellular origin are generated by epithelial cells and originate in the epidermis' deeper layer, the dermis' and from the hair follicles root sheath. Human cellular origin was confirmed by histological investigations, which revealed that these filaments are made mostly of keratin and collagen, and are nucleated at the base of attachment to epithelial cells [35].

On Electron microscopy hair like scales were observed on blue filament, indicating that some fibers of the disease are hair like in structure [36]. Figure 4 shows positively stained filaments which reveal basal layer origin [5]. Fontana Masson staining positive result revealed blue color pigment in fibers due to the melanin production [35]. 


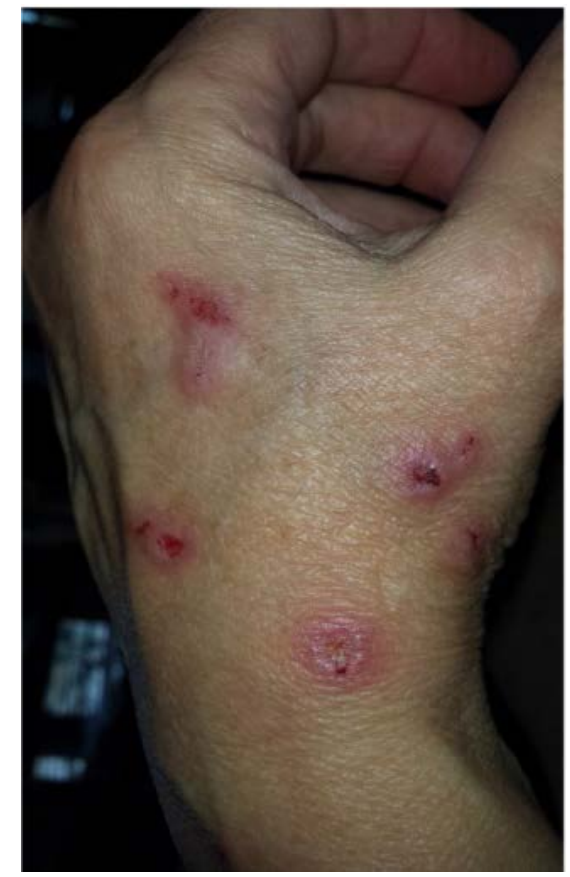

(a)

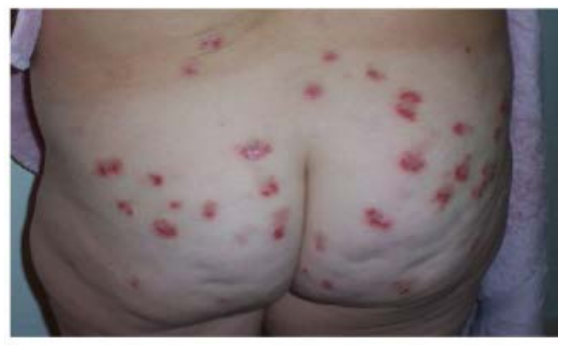

(b)

Figure 3. (a) Morgellons disease lesion on hand. (b) Skin lesion on buttocks (Middelveen, M.J., et al., 2016) [7].
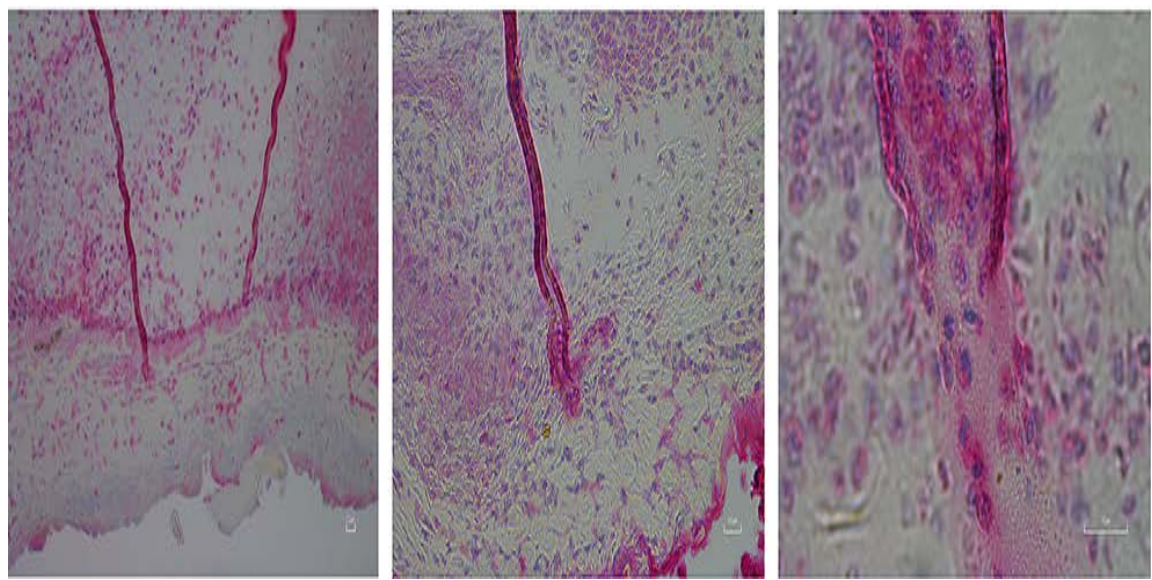

Figure 4. Positively stained filaments revealing basal cell layer origin (Middelveen, M.J. et al., 2020) [5].

\section{Molecular Diagnosis:}

Borrelia DNA was identified by polymerase chain reaction (PCR) and Sanger sequencing. Motile spirochetes i.e., Borrelia species were cultured in Barbour-Stoenner-Kelly (BSK)-H medium and inoculated with Morgellons disease skin tissue. The presence and molecular identification of live Borrelia spirochetes in Morgellons disease skin specimen was confirmed by PCR amplification of cultured spirochetes. Borrelia culture and PCR altogether provide significant evidence for a clinical link between spirochetal infection and Morgellons disease [7]. 


\subsection{Treatment}

Because a clinical categorization for Morgellons disease has yet to be agreed upon, the best treatment for the condition is unknown. Several therapeutic concepts have evolved.

1) The earlier the treatment begins in the course of morgellons disease, the better the outcome seems to be.

2) The therapy should target the underlying tick-borne disease.

3) Prolonged antibiotic combination therapy may be required to eliminate dermopathy.

4) Antiparasitic medication may be beneficial in some Morgellons disease patients [37].

5) Patients with neuropsychiatric illness have been suggested to be treated with antipsychotic drugs [38].

\section{Conclusion}

Piscine mycobacteriosis is a zoonotic illness that affects both freshwater and marine fish globally. The etiological factor for piscine mycobacteriosis is Mycobacterium species; more than 20 strains of Mycobacterium species have been found in fish. Humans get infected by Mycobacterium marinum via contaminated water. Mycobacterium marinum is an opportunistic infection in fishes its control prophylactically and prevention by vaccination is the need of hour and because of the communicable nature of the disease, precautions should be taken i.e., by avoiding swimming in fish pools and using gloves while handling and preparing fish. While on the other hand Morgellons disease is a new kind of dermopathy linked to Borrelia infection, and the rising number of Morgellons disease patients parallels the global rise in tick-borne illnesses. Sometimes Medical practitioners misdiagnose it as a delusional disorder due to the false idea that the fibers are generated from textiles. According to the previous studies Morgellons disease is a somatic disease that seems to be induced by Borrelia infection. The best therapy for this illness has yet to be discovered. Thus, on the basis of the available data, it can be concluded that there is no evidence of correlation between Mycobacterium marinum and Morgellons disease. Further research is required to understand the disease process of these complex entities and to evaluate the best therapy for both of the diseases.

\section{Conflicts of Interest}

The authors declare no conflicts of interest regarding the publication of this paper.

\section{References}

[1] Keller. C., Wenker, C., Jermann, T., Hirschi, R., Schildger, B., Meie, R., et al. (2018) Piscine Mycobacteriosis-Involvement of Bacterial Species and Reflection in $\mathrm{Pa}$ thology. Schweiz Arch Tierheilkd, 160, 385-393. https://doi.org/10.17236/sat00165 
[2] Decostere. A., Hermans. K. and Haesebrouck, F. (2004) Piscine Mycobacteriosis: A Literature Review Covering the Agent and the Disease It Causes in Fish and $\mathrm{Hu}-$ mans. Veterinary Microbiology, 99, 159-166. https://doi.org/10.1016/j.vetmic.2003.07.011

[3] Hashish, E., Merwad, A., Elgaml, S., Amer, A., Kamal, H., Elsadek, A., et al. (2018) Mycobacterium marinum Infection in Fish and Man: Epidemiology, Pathophysiology and Management; A Review. Veterinary Quarterly, 38, 35-46. https://doi.org/10.1080/01652176.2018.1447171

[4] Akram, S.M. and Aboobacker, S. (2021) Mycobacterium Marinum. StatPearls Publishing, Treasure Island. https://www.ncbi.nlm.nih.gov/books/NBK441883/

[5] Middelveen, M.J., Martinez, R.M., Fesler, M.C., Sapi, E., Burke, J., Shah, J.S., et al. (2020) Classification and Staging of Morgellons Disease: Lessons from Syphilis. Clinical, Cosmetic and Investigational Dermatology, 13, 145-164. https://doi.org/10.2147/CCID.S239840

[6] Middelveen, M.J., Filush, K.R., Bandoski, C., Kasliwala, R.S., Melillo, A., Stricker, R.B., et al. (2019) Mixed Borrelia burgdorferi and Helicobacter pylori Biofilms in Morgellons Disease Dermatological Specimens. Healthcare, 7, Article No. 70. https://doi.org/10.3390/healthcare7020070

[7] Middelveen, M.J. and Stricker, R.B. (2016) Morgellons Disease: A Filamentous Borrelial Dermatitis. International Journal of General Medicine, 9, 349-354. https://doi.org/10.2147/IJGM.S116608

[8] Jacobs, J.M., Stine, C.B., Baya, A.M. and Kent, M.L (2009) A Review of Mycobacteriosis in Marine Fish. Journal of Fish Diseases, 32, 119-130.

https://doi.org/10.1111/j.1365-2761.2008.01016.x

[9] Gauthier, D. T. and Rhodes, M.W. (2009) Mycobacteriosis in Fishes: A Review. The Veterinary Journal, 180, 33-47. https://doi.org/10.1016/j.tvjl.2008.05.012

[10] Grange, J.M. (1981) Mycobacterium chelonei. Tubercle, 62, 273-276. https://doi.org/10.1016/S0041-3879(81)80007-4

[11] Ross, A.J. and Brancato, F.P. (1959) Mycobacterium Fortuitum Cruz from the Tropical fish Hyphessobrycon innesi. Journal of Bacteriology, 78, 392-395. https://doi.org/10.1128/jb.78.3.392-395.1959

[12] Janse, M. and Kik, M.J.L (2012) Mycobacterium avium Granulomas in a Captive Epaulette Shark, Hemiscyllium ocellatum (Bonnaterre). Journal of Fish Diseases, 35, 935-940. https://doi.org/10.1111/j.1365-2761.2012.01444.x

[13] Fukano, H., Wada, S., Kurata, O., Katayama, K., Fujiwara, N. and Hoshino, Y. (2017) Mycobacterium stephanolepidis sp. Nov., a Rapidly Growing Species Related to Mycobacterium chelonae, Isolated from Marine Teleost Fish, Stephanolepis cirrhifer. International Journal of Systematic and Evolutionary Microbiology, 67, 2811-2817. https://doi.org/10.1099/ijsem.0.002028

[14] Delghandi, M.R., El-Matbouli, M. and Menanteau-Ledouble, S. (2020) Mycobacteriosis and Infections with Non-Tuberculous Mycobacteria in Aquatic Organisms: A Review. Microorganisms, 8, Article No. 1368. https://doi.org/10.3390/microorganisms8091368

[15] Passantino, A., Macrì, D., Coluccio, P., Foti, F. and Marino, F. (2008) Importation of Mycobacteriosis with Ornamental Fish: Medico-Legal Implications. Travel Medicine and Infectious Disease, 6, 240-244. https://doi.org/10.1016/j.tmaid.2007.12.003

[16] Bhatty, M.A., Turner, D.P.J. and Chamberlain, S.T. (2000) Mycobacterium marinum Hand Infection: Case Reports and Review of Literature. British Journal of Plastic Surgery, 53, 161-165. https://doi.org/10.1054/bjps.1999.3245 
[17] Dusthackeer, A., Balasubramanian, M., Shanmugam, G., Priya, S., Nirmal, C.R., Sam Ebenezer, R., Balasubramanian, A., Mondal, R.K., Thiruvenkadam, K., Hemanth Kumar, A.K., Ramachandran, G. and Subbian, S. (2019) Differential Culturability of Mycobacterium tuberculosis in Culture-Negative Sputum of Patients With Pulmonary Tuberculosis and in a Simulated Model of Dormancy. Frontiers in Microbiology, 10, Article No. 2381. https://doi.org/10.3389/fmicb.2019.02381

[18] Bonamonte, D., De Vito, D., Vestita, M., Delvecchio, S., Ranieri, L.D., Santantonio, M., et al. (2013) Aquarium-Borne Mycobacterium marinum Skin Infection. Report of 15 Cases and Review of the Literature. European Journal of Dermatology, 23, 510-516. https://doi.org/10.1684/ejd.2013.2103

[19] Rhodes, M.W., Kator, H., McNabb, A., Deshayes, C., Reyrat, J.-M., et al. (2005) Mycobacterium pseudoshottsii sp. nov., a Slowly Growing Chromogenic Species Isolated from Chesapeake Bay Striped Bass (Morone saxatilis). International Journal of Systematic and Evolutionary Microbiology, 55, 1139-1147.

https://doi.org/10.1099/ijs.0.63343-0

[20] Whipps, C.M., Butler, W.R., Pourahmad, F., Watral, V.G. and Kent, M.L. (2007) Molecular Systematics Support the Revival of Mycobacterium salmoniphilum (ex Ross 1960) sp. nov., nom. rev., a Species Closely Related to Mycobacterium chelonae. International Journal of Systematic and Evolutionary Microbiology, 57, 2525-2531. https://doi.org/10.1099/ijs.0.64841-0

[21] Whipps, C.M., Dougan, S.T. and Kent, M.L. (2007) Mycobacterium haemophilum Infections of Zebrafish (Danio rerio) in Research Facilities. FEMS Microbiology Letters, 270, 21-26. https://doi.org/10.1111/j.1574-6968.2007.00671.x

[22] Talaat, A.M., Reimschuessel, R. and Trucksis, M. (1997) Identification of Mycobacteria Infecting Fish to the Species Level Using Polymerase Chain Reaction and Restriction Enzyme Analysis. Veterinary Microbiology, 58, 229-237.

https://doi.org/10.1016/S0378-1135(97)00120-X

[23] Chemlal, K. and Portaels, F. (2003) Molecular Diagnosis of Nontuberculous Mycobacteria. Current Opinion in Infectious Diseases, 16, 77-83.

https://doi.org/10.1097/00001432-200304000-00003

[24] Turenne, C.Y., Tschetter, L., Wolfe, J. and Kabani, A. (2001) Necessity of Quality-Controlled 16S rRNA Gene Sequence Databases: Identifying Nontuberculous Mycobacterium Species. Journal of Clinical Microbiology, 39, 3637-3648. https://doi.org/10.1128/JCM.39.10.3638-3648.2001

[25] Poort, M.J., Whipps, C.M., Watral, V.G., Font, W.F. and Kent, M.L. (2006) Molecular Characterization of a Mycobacterium Species in Non-Native Poeciliids in Hawaii Using DNA Sequences. Journal of Fish Diseases, 29, 181-185. https://doi.org/10.1111/j.1365-2761.2006.00687.x

[26] Salati, F., Meloni, M., Fenza, A., Angelucci, G., Colorni, A. and Orrù, G. (2010) A Sensitive FRET Probe Assay for the Selective Detection of Mycobacterium marinum in Fish. Journal of Fish Diseases, 33, 47-56. https://doi.org/10.1111/j.1365-2761.2009.01112.x

[27] Kusuda, R. and Kawakami, K. (1990) Efficacy of Rifampicin, Streptomycin and Erythromycin against Experimental Mycobacterium Infection in Cultured Yellowtail. Nippon Suisan Gakkaishi, 56, 51-53. https://doi.org/10.2331/suisan.56.51

[28] Chang, C.T. and Whipps, C.M. (2015) Activity of Antibiotics against Mycobacterium Species Commonly Found in Laboratory Zebrafish. Journal of Aquatic Animal Health, 27, 88-95. https://doi.org/10.1080/08997659.2015.1007176

[29] Kato, G., Kondo, H., Aoki, T. and Hirono, I. (2010) BCG Vaccine Confers Adaptive 
Immunity against Mycobacterium sp. Infection in Fish. Developmental \& Comparative Immunology, 34, 133-140. https://doi.org/10.1016/j.dci.2009.08.013

[30] Ziklo, N., Colorni, A., Gao, L.Y., Du, S.J. and Ucko, M. (2018) Humoral and Cellular Immune Response of European Seabass Dicentrarchus labrax Vaccinated with Heat-Killed Mycobacterium marinum (iipA::kan Mutant). Journal of Aquatic Animal Health, 30, 312-324. https://doi.org/10.1002/aah.10042

[31] Myllymäki, H., Niskanen, M., Oksanen, K.E., Sherwood, E., Ahava, M., Parikka, M., et al. (2017) Identification of Novel Antigen Candidates for a Tuberculosis Vaccine in the Adult Zebrafish (Danio rerio). PLoS ONE, 12, Article ID: e0181942. https://doi.org/10.1371/journal.pone.0181942

[32] Kellett C.E. (1935) Sir Thomas Browne and the Disease Called the Morgellons. Annals of Medical History, 7, 467-479.

https://www.ncbi.nlm.nih.gov/pmc/articles/PMC7942326/

[33] Savely, V.R., Leitao, M.M. and Stricker, R.B. (2006) The Mystery of Morgellons Disease: Infection or Delusion? American Journal of Clinical Dermatology, 7, 1-5. https://doi.org/10.2165/00128071-200607010-00001

[34] Pearson, M.L., Selby, J.V., Katz, K.A., Cantrell, V., Braden, C.R., Parise, M.E., et al. (2012) Clinical, Epidemiologic, Histopathologic and Molecular Features of an Unexplained Dermopathy. PLoS ONE, 7, Article ID: e29908. https://doi.org/10.1371/journal.pone.0029908

[35] Middelveen, M., Mayne, P., Kahn, D. and Stricker, R. (2013) Characterization and Evolution of Dermal Filaments from Patients with Morgellons Disease. Clinical, Cosmetic and Investigational Dermatology, 6, 1-21. https://doi.org/10.2147/CCID.S39017

[36] Middelveen, M.J., Rasmussen, E.H., Kahn, D.G. and Stricker, R.B. (2012) Morgellons Disease: A Chemical and Light Microscopic Study. Journal of Clinical \& Experimental Dermatology Research, 3, Article No. 140. https://doi.org/10.4172/2155-9554.1000140

[37] Cameron, D.J., Johnson, L.B. and Maloney, E.L. (2014) Evidence Assessments and Guideline Recommendations in Lyme Disease: The Clinical Management of Known Tick Bites, Erythema Migrans Rashes and Persistent Disease. Expert Review of Anti-Infective Therapy, 12, 1103-1135. https://doi.org/10.1586/14787210.2014.940900

[38] Mayne, P., English, J.S., Kilbane, E.J., Burke, J.M., Middelveen, M.J. and Stricker, R.B. (2013) Morgellons: A Novel Dermatological Perspective as the Multisystem Infective Disease Borreliosis. F1000Research, 2, Article No. 118. https://doi.org/10.12688/f1000research.2-118.v1 